\title{
Medical management of ankylosing spondylitis
}

\author{
Alexander A. Khalessi, M.D., M.S., ${ }^{1}$ Bryan C. OH, M.D., ${ }^{1}$ And Michael Y. Wang, M.D. ${ }^{2}$ \\ ${ }^{\prime}$ Department of Neurological Surgery, University of Southern California, Los Angeles, California; \\ and ${ }^{2}$ Department of Neurological Surgery, University of Miami, Florida
}

\begin{abstract}
$\checkmark$ In the following literature review the authors consider the available evidence for the medical management of patients with ankylosing spondylitis (AS), and they critically assess current treatment guidelines. Medical therapy for axial disease in AS emphasizes improvement in patients' pain and overall function. First-line treatments include individualized physical therapy and nonsteroidal antiinflammatory drugs (NSAIDs) in conjunction with gastroprotective therapy. After an adequate trial of therapy with two NSAIDs exceeding 3 months or limited by medication toxicity, the patient may undergo tumor necrosis factor- $\alpha$ blockade therapy. Response should occur within 6-12 weeks, and patients must undergo tuberculosis screening. Evidence does not currently support the use of disease modifying antirheumatic drugs, corticosteroids, or radiotherapy in AS. (DOI: 10.3171/FOC/2008/24/1/E4)
\end{abstract}

\section{KEY WORDS • ankylosing spondylitis nonsteroidal antiinflammatory drug \\ - medical management • \\ tumor necrosis factor $-\alpha$ blockade}

A NKYLOSING spondylitis is a chronic, inflammatory rheumatic disease. Its pathogenesis involves the formation of syndesmophytes with resultant spinal ankylosis. The vertebral column transforms from a dynamic structure with ligamentous attachments into a rigid column of tubular bone housing the spinal cord and neural elements. Sacroiliitis, spondylitis, and enthesitis all contribute to characteristic inflammatory axial pain. Common extraspinal manifestations of AS include peripheral arthritis (25-50\%), uveitis (25-40\%), inflammatory bowel disease $(26 \%)$, and psoriasis $(10 \%){ }^{14}$

Genetic studies have demonstrated a strong association with the HLA-B27 gene with a resultant preponderance for populations at northern latitudes. ${ }^{8}$ The authors of recent epidemiological studies have estimated the prevalence rates to range from $0.4 \%$ among Alaskan Eskimos to $1.4 \%$ in Norway. ${ }^{8}$ Notably, men are 2 to 3 times more likely than women to suffer from AS. Peak age of onset is between 20-30 years, with an average 5-6-year delay in diagnosis reported in the literature. ${ }^{15}$

The disease course, although highly variable, will progress to severe disability in one third of patients. Manifestations of AS symptoms include back pain and stiffness in adolescence and early adulthood, and impaired chest wall expansion and spinal mobility may result. Patients

Abbreviations used in this paper: AS = ankylosing spondylitis; ASAS = Assessment in Ankylosing Spondylitis; BASDAI = Bath Ankylosing Spondylitis Disease Activity Index; BASFI = BAS Functional Index; $\mathrm{CI}=$ confidence interval; DMARD $=$ diseasemodifying antirheumatic drug; ES = effect size; EULAR = European League Against Rheumatism; NSAID = nonsteroidal antiinflammatory drug; $\mathrm{RR}=$ relative risk; $\mathrm{TNF}=$ tumor necrosis factor. with AS experience higher work disability and absenteeism than those with rheumatoid arthritis and thereby suffer substantial direct and indirect economic costs. ${ }^{16}$

Treatment goals in AS center around 3 central principles: 1) symptom relief, 2) functionality relative to activities of daily living and work performance, and 3) delay or avoidance of the structural damage responsible for physical impairments or deformity. To this end, the ASAS International Working Group, in collaboration with the EULAR, developed consensus guidelines for the medical management of AS. Ten propositions, ranging from diagnosis to physical therapy and medical management, emerged from a 3-round Delphi exercise from a panel of experts; heavy consideration was given to supporting literature for various treatment modalities. ${ }^{15}$

In the following review we summarize the ASAS/ EULAR consensus recommendations and include a systematic discussion of relevant studies. Discussion will proceed from general considerations in AS, namely diagnosis and disease monitoring, to nonpharmacological management tools and available medications. Surgical management of AS will be discussed elsewhere.

\section{Medical Management of Ankylosing Spondylitis}

Outcomes Assessment: Patient Diagnosis and Disease Monitoring

Assessment of patients with AS represents a multifactorial challenge in terms of systemic manifestations (axial, peripheral, etheseal, and extraarticular symptoms and signs) and longitudinal variation (active disease versus disease remission). Disease monitoring requires meticulous clinical history, laboratory serum studies, and imaging 
modalities. Given the ASAS/EULAR therapeutic goals of symptom relief and enhanced functionality, outcome assessment and treatment considerations center on criteria for initial diagnosis and the definition of active disease. ${ }^{2}$

New York Criteria. The modified New York criteria serve as the basis for the ASAS/EULAR recommendations. Although mildly restrictive in its definition of AS, the radiographic presence of sacroiliitis is a necessary condition for diagnosis. Using plain x-ray films, the severity of sacroiliac joint disease is divided into 4 grades. Grades I and II represent initial joint space widening, followed by joint space narrowing and the indistinctness of the sacroiliac joint. Grades III and IV involve bone destruction and ultimate fusion of the sacroiliac joint. The presence of a grade $\geq$ II bilaterally or Grade III or IV unilaterally satisfies the New York criteria for the diagnosis of AS.

Aside from sacroiliitis, the patient must exhibit 2 of the following 3 clinical criteria for a diagnosis of AS to be established: 1) low-back pain and stiffness $\geq 3$ months with palliation by exercise and no relief with rest; 2) limitation of lumbar spinal motion in sagittal and coronal planes; and 3) limitation of chest expansion relative to ageand sex-matched individuals. Although the limitations of the New York criteria include their conservative reliance on a sacroiliitis presentation and may exclude other dimensions of AS, the overwhelming majority of randomized controlled trials involving AS rely on these criteria. ${ }^{16}$

\section{Disease Monitoring}

Disease activity encompasses 3 fundamental aspects: 1) persistence of a patient's symptomatology, 2) threat of severe disease and structural damage, and 3) response to treatment. The BASFI and BASDAI represent validated survey instruments by which to assess disease activity from the patient's perspective. The BASFI includes 10 binary questions regarding the completion of activities of daily living. The BASDAI, by contrast, utilizes a visual analog scale to quantify a patient's overall fatigue, axial pain, joint swelling, tenderness, stiffness, and symptom duration. Both the BASFI and BASDAI serve as important treatment endpoints in the rheumatology literature; failure to improve along these parameters often justifies advancement to more aggressive therapies. ${ }^{14}$

Accepted serum markers for disease staging include Creactive protein and erythrocyte sedimentation rate. Antinuclear antibodies and liver function tests are important in patients undergoing immunological therapy. Radiographically documented progression on sacroiliac or lateral spinal x-ray films provides further insight into disease activity and structural damage. ${ }^{3}$ Contrast-enhanced magnetic resonance imaging, especially in the spine, is gaining wider acceptance as a snapshot reflection of ongoing inflammation. The roles of ultrasonography, nuclear medicine, and bone scanning studies remain unclear and of limited utility at present.

Notably, a distinction should be made in patients with mechanical back pain or pathological spinal fusion that precipitates mechanical restriction or disability. Medical therapy targets the inflammatory component of AS pathogenesis. Thus, mechanical complaints in patients with known spinal deformity should be distilled from BASDAI and BASFI results in consideration of medical therapies.
Having thereby defined the AS disease state and its severity over time, one may consider nonpharmacological and pharmacological therapies. The ES, defined by mean change divided by standard deviation of the change, and $95 \%$ CIs are included where available. ${ }^{16}$

\section{Physical Therapy and Education}

Members of the Cochrane Collaboration undertook a systematic review of physiotherapy interventions for AS. The metaanalysis included 6 trials involving 561 patients. Kraag et al. ${ }^{9}$ have reported that an individual therapeutic exercise program significantly improved functionality at four months (ES 1.14, 95\% CI 0.55-1.73) but did not provide pain relief. Hidding et al. ${ }^{7}$ confirmed enhanced patient assessments of functionality and objective spinal mobility $(p<0.05)$ with physical therapy. Supervised group therapy, however, did not confer a benefit beyond individual home regimens. A third physiotherapy trial involving a composite outcome measure for pain and functionality did not establish a benefit. More recently, a targeted study of young (mean age 28 years), previously sedentary AS patients showed significant 8-week gains in both pain relief (ES 1.99, 95\% CI 1.30-2.67) and function (ES 0.80, 95\% CI 0.23-1.38). ${ }^{11}$ Level Ib evidence therefore supports the use of individual physiotherapy as a primary treatment for axial AS; younger patients with low baseline activity levels stand to benefit the most from physical therapy interventions. ${ }^{6}$

Data regarding adjuvant therapies remain sparse and largely unsupportive. Although a Level IV study found that a 3-week spa program combined with physical therapy enhanced overall patient well-being and achieved shortterm gains, no significant additive benefit in terms of pain or functionality was realized at 40 weeks. Transcutaneous electrical nerve stimulation versus sham stimulation over 3 weeks barely failed to reach significance in terms of pain relief ( $p=0.05$ ); the ES, however, was also small (ES 0.92). Heat and whole-body cryotherapy studies also did not show benefits to AS patients. ${ }^{16}$

In terms of education programs, the authors of a controlled study found that self-management courses failed to improve pain and functionality at 6 months but did improve patient self-sufficiency and motivation. ${ }^{12}$ Cognitive behavioral therapy further showed a convincing improvement in anxiety (ES 1.11, 95\% CI 0.48-1.75). The authors asserted that indirect cost savings from a reduction in work days lost compensate for educational expenses. Education may therefore diminish the psychiatric and affective comorbidities associated with chronic diseases such as AS. ${ }^{16}$

Lifestyle modification data remain equivocal. Smoking cessation may confer some benefit; 3 cross-sectional studies demonstrated poorer functional outcomes in patients with AS who smoke. ${ }^{18}$ Open interventional trials regarding dietary restriction of starch or dairy foods remain anecdotal at this time. ${ }^{6}$

\section{Nonsteroidal Antiinflammatory Drugs, Coxibs, and Corticosteroids}

Eight randomized trials support the use of NSAIDs or coxibs for pain in patients with AS. ${ }^{16}$ Level Ib evidence indicates that NSAIDs improve spinal pain compared with placebo (ES 1.11, 95\% CI 0.96-1.26) and function (ES 
$0.62,95 \%$ CI $0.26-0.97)$ over a 6-week period. Coxibs exhibit comparable efficacy for spinal pain (ES 1.05, $95 \%$ CI $0.88-1.22$ ) and for function (ES 0.63 , 95\% CI 0.26 0.97). Notably, AS patients with chronic peripheral arthritis may exhibit poorer spinal response rates to NSAIDs and coxibs; experience with etoricoxib suggests this phenomenon. ${ }^{16}$ In this drug class, no existing trial supports the use of a particular agent over another.

Given the use of NSAIDs as primary pharmacological therapy, advancement to more aggressive medical regimens requires an adequate therapeutic trial. The ASAS/ EULAR authors defined NSAID trial adequacy as at least 2 NSAIDs administered 1) at the maximal recommended or tolerated antiinflammatory dose for $\geq 3$ months or 2 ) $<$ 3 months when NSAID cessation corresponded with intolerance toxicity or contraindications. Although beyond the scope of AS axial disease, conservative treatment of severe peripheral arthritis requires a trial of sulfasalazine; local symptoms from enthesitis require adjuvant corticosteroid injections..$^{5}$ Later consideration of these medical therapies will establish their failure to treat or modify AS spinal disease.

Nonsteroidal antiinflammatory drug and coxib toxicity profiles center around gastrointestinal and cardiovascular complications. Nonsteroidal antiinflammatory drugs confer a dose-dependent increased risk of gastrointestinal bleeding (RR 5.36, 95\% CI 1.79-16.10). Gastroprotective agents such as misoprostol, $\mathrm{H} 2$ blockers, and proton-pump inhibitors mitigate this risk (RR $0.40,95 \%$ CI $0.03-0.74$ ). Coxibs pose a lower overall risk of serious gastrointestinal toxicity but are associated with considerable diarrhea and dyspeptic symptoms. Coxibs further carry an increased risk of thromboembolic complications such as myocardial infarction or stroke. Relative risks of rofecoxib 2.30 (95\% CI 1.22-4.33), valdecoxib 3.7 (95\% CI 1.0-13.5) and naproxen 2.0 (no CI available) bear out this class effect. Data regarding the cardiovascular complications of NSAID use are under investigation. ${ }^{15}$

According to the ASAS/EULAR guidelines, NSAIDs are therefore the first-line drug treatment for AS patients with pain and stiffness. In the face of increased gastrointestinal risks, either a nonselective NSAID plus a gastroprotective agent, or a selective cyclooxygenase-2 inhibitor should be used. Evidence does not currently support the use of local or systemic corticosteroid therapy in patients with AS spinal disease. Class Ib evidence exists that local corticosteroid injections may provide short-term palliation of sacroiliitis symptoms (ES 1.92, 95\% CI 0.53-3.35) and Class IV evidence supports local steroid injections for enthesitis. ${ }^{16}$

\section{Disease-Modifying Antirheumatic Drugs}

Sulfasalazine and methotrexate represent the most widely used DMARDs. Individual Cochrane reviews exist regarding the utility of these agents in the treatment of AS. Unfortunately, neither agent meaningfully modifies pain or functionality attributable to AS spinal disease., ${ }^{4,5}$

Eleven randomized trials examined sulfasalazine use in patients with AS and contributed to the Cochrane metaanalysis. ${ }^{16}$ Although erythrocyte sedimentation rate (mean $-4.79,95 \%$ CI -8.80 to -0.78 ) and morning stiffness (-13.89 on a 100 -point visual analog scale; $95 \%$ CI -22.54 to -5.24) improved significantly with sulfasalazine, back pain (ES $-2.38,95 \% \mathrm{CI}-5.78$ to 1.03 ) and physical function (ES $0.20,95 \% \mathrm{CI}-0.77$ to 1.18 ) did not improve. Moreover, common toxicities (RR 2.37 for adverse event, 95\% CI 1.58-3.55) include gastrointestinal symptoms, mucocutaneous, hepatic, or hematological manifestations. ${ }^{13}$

The Cochrane review of methotrexate examined 116 patients with AS across 3 trials and found no significant improvements in BASDAI or BASFI. Pooled results for spinal pain ( $\mathrm{ES}-0.05,95 \% \mathrm{CI}-0.48$ to 0.38 ) and function (ES $0.02,95 \%$ CI -0.40 to 0.45 ) failed to achieve statistical significance relative to placebo. Methotrexate toxicities included nausea (RR 2.12,95\% CI 1.50-2.98) and hepatic abnormalities (RR 4.12, 95\% CI 2.22-7.63). In an observational study of 14 patients, the authors estimated that $21 \%$ of patients taking methotrexate must stop the drug due to toxicity. ${ }^{4}$

Bisphosphonates and thalidomide remain other areas of open DMARD investigation in AS. Maksymowych et al. ${ }^{14}$ published a Class III study involving high-dose pamidronate and reported significantly improved function (ES $0.73,95 \%$ CI $0.29-1.17)$ and axial pain $(\mathrm{p}=0.003)$. Anecdotal evidence supports the use of thalidomide for axial pain, but current ASAS/EULAR guidelines found thalidomide's toxicity profile prohibitive. ${ }^{15}$

The DMARDs, including sulfasalazine and methotrexate, therefore do not currently enjoy empirical support for their use in axial AS disease. Sulfasalazine may have a limited role in treatment of peripheral joint symptoms. ${ }^{5}$

\section{Tumor Necrosis Factor and Interleukin Inhibitors}

In 1995, Braun ${ }^{1}$ isolated TNF $\alpha$ from the sacroiliac aspirates obtained in patients with AS. Given the known prominence of $\mathrm{TNF} \alpha$ in the inflammatory cascade, biological agents were developed to target TNF $\alpha$ activity. Three agents are currently available: 1) infliximab (Remicaide), a chimeric, murine-human monoclonal immunoglobulin $\mathrm{G}$ antibody that binds TNF $\alpha$ with high affinity; 2) etanercept (Enbrel), a receptor fusion protein that competitively binds TNF $\alpha$ binding and activation at the cell membrane; and 3) adalimumab (Humira), a recombinant, purely human monoclonal immunoglobulin $\mathrm{G}$ antibody to TNF $\alpha .{ }^{2}$

Level Ib evidence strongly supports the use of etanercept and infliximab for spinal pain, function, and peripheral joint disease. Large ESs, low numbers-needed-to-treat, and broad class benefits underscore the strength of TNF $\alpha$ inhibitor benefit. Across 2 randomized trials, etanercept demonstrated a spinal pain and function ES of 2.25 (95\% CI 1.92-2.59) and 2.11 (95\% CI 1.81-2.41), respectively. ${ }^{11}$ The number needed-to-treat to observe significant benefit versus placebo was only 2.7 (95\% CI 2.2-3.4). Infliximab offered comparable results: spinal pain and function ESs were 0.90 (95\% CI 0.66-1.14) and 0.93 (CI 0.69-1.17), respectively, and the number-needed-to-treat was $2.3(95 \%$ CI 1.8-2.4). Notably, only one Level III trial exists for the newer adalimumab, but comparable class benefits were observed. ${ }^{16}$

Moreover, the onset of therapeutic benefit with $\mathrm{TNF} \alpha$ inhibitors is rapid, and the therapeutic effect persists for 3 years with continuing treatment. Treatment cessation results in a high incidence of relapse. Due to the rapid onset, ASAS/EULAR established a 6-12-week time frame to 
identify nonresponders and terminate therapy. ${ }^{15}$ The need for rapid identification of patients with AS not experiencing the benefits of TNF $\alpha$ blockade is to spare the patient any therapeutic risks. ${ }^{14}$

The TNF $\alpha$ inhibition increases the patient's susceptibility to common upper respiratory infections and tuberculous disease; screening for Mycobacterium tuberculosis is now a prerequisite for therapy initiation. Infliximab may precipitate a positive antinuclear antibody titer, and the incidences of demyelinating disease and lupuslike syndromes, although anecdotally reported, remains unknown. ${ }^{2}$

\section{Radiation Therapy}

Local irradiation of the spine and sacroiliac joints may provide pain relief for up to 12 months (Level Ib evidence). However, the carcinogenicity of this treatment, namely for leukemia (RR 2.74, 95\% CI 2.10-3.53) and other malignancies (RR 1.26, 95\% CI 1.19-1.32), precludes its widespread use. ${ }^{16}$

\section{Conclusions}

The goals of medical therapy for axial disease in AS are improvement in pain and overall function. Initial diagnosis centers on the presence of sacroiliitis coupled with characteristic axial pain and stiffness $\geq 3$ months, loss of spinal motion, or restricted chest wall expansion $<2.5 \mathrm{~cm}$. The initiation of treatment follows the satisfaction of the enumerated New York criteria for AS.

First-line treatments include individualized physical therapy and administration of NSAIDs in conjunction with gastroprotective therapy. After an adequate trial of 2 NSAIDs $>3$ months or limited by medication toxicity, the patient may undergo TNF $\alpha$ blockade therapy. A response should occur within 6 to 12 weeks, and patients must undergo screening for tuberculosis. Evidence does not currently support the use of DMARDs, corticosteroids, or radiotherapy in AS.

Active areas of inquiry include TNF $\alpha$ blockade analogs and genetic twin and cohort studies to characterize the inheritance and environmental precipitants of AS.

\section{References}

1. Braun J, Bollow M, Neure L, Seipelt E, Seyrekbasan F, Herbst H, et al: Use of immunohistochemical and in situ hybridization techniques in the examination of sacroiliac joint biopsy specimens from patients with ankylosing spondylitis. Arthritis Rheumat 38:499-505, 1995

2. Braun J, Pham T, Sieper J, Davis J, van deer Linden SJ, Dougados $\mathrm{M}$, et al: International ASAS consensus statement for the use of anti-tumor necrosis factor in agents with ankylosing spondylitis. Ann Rheum Dis 62:817-824, 2003

3. Braun J, Sieper J: Ankylosing spondylitis. Lancet 369:13791390, 2007

4. Chen J, Liu C: Sulfasalazine for ankylosing spondylitis. Cochrane Database Syst Rev 2: CD004800, 2002

5. Chen J, Liu C, Lin J: Methotrexate for ankylosing spondylitis. Cochrane Database Syst Rev 4: CD004524, 2006

6. Dagfinrud H, Hagen KB: Physiotherapy interventions for ankylosing spondylitis. Cochrane Database Syst Rev 4: CD002822, 2001

7. Hidding A, van der Linden S, Boers M, Gielen X, de Witte L, Kester A, et al: Is group physical therapy superior to individualized therapy in ankylosing spondylitis? A randomized controlled trial. Arthritis Care Res 6:117-125, 1993

8. Khan MA: Update on spondyloarthropathies. Ann Intern Med 135:896-907, 2002

9. Kraag G, Stokes B, Groh J, Helewa A, Goldsmith C: The effects of comprehensive home physiotherapy and supervision on patients with ankylosing spondylitis-a randomized controlled trial. J Rheumatol 17:228-233, 1990

10. Krauth C, Rieger J, Bönisch A, Ehlebracht-König I: [Costs and benefits of an education program for patients with ankylosing spondylitis as part of an inpatient rehabilitation program - study design and first results.] Z Rheumatol 62:114-116, 2003 (Ger)

11. Lim HJ, Moon Y, Lee MS: Effects of home-based daily exercise therapy on joint mobility, daily activity, pain, and depression in patients with ankylosing spondylitis. Rheumatol Int 25:225-229, 2005

12. Maksymowych WP, Jhangri GS, Fitzgerald AA, LeClerq S, Chiu P, Yan A, et al: A six-month randomized, controlled, doubleblind, dose-response comparison of intravenous pamidronate (60 $\mathrm{mg}$ versus $10 \mathrm{mg}$ ) in the treatment of nonsteroidal antiinflammatory drug-refractory ankylosing spondylitis. Arthritis Rheumatol 46:766-773, 2002

13. Ward MM, Kuzis S: Medication toxicity among patients with ankylosing spondylitis. Arthritis Rheum 47:234-241, 2002

14. Zochling J, Maxwell L, Beardmore J, Boonen A: TNF-alpha inhibitors for ankylosing spondylitis. Cochrane Database Systemic Rev 3: CD005468, 2005

15. Zochling J, van der Heijde D, Burgos-Vargas R, Collantes E, Davis JC Jr, Dijkmans B, et al: ASAS/EULAR recommendations for the management of ankylosing spondylitis. Ann Rheum Dis 65:442-452, 2006

16. Zochling J, van der Heijde D, Dougados M, Braun J: Current evidence for the management of ankylosing spondylitis: a systemic literature review of the ASAS/EULAR management recommendations in ankylosing spondylitis. Ann Rheum Dis 65:423-432, 2006

Manuscript submitted October 15, 2007.

Accepted November 27, 2007.

Address correspondence to: Alexander A. Khalessi, M.D., Department of Neurological Surgery, University of Southern California, Los Angeles, California. email: khalessi@usc.edu. 